\title{
Erratum to: Surface expression and channel function of TRPM8 are cooperatively controlled by transmembrane segments $\mathrm{S3}$ and $\mathrm{S} 4$
}

\author{
Frank J. P. Kühn • Mathis Winking • Cornelia Kühn • \\ Daniel C. Hoffmann • Andreas Lückhoff
}

Published online: 26 July 2013

(C) Springer-Verlag Berlin Heidelberg 2013

\section{Erratum to: Pflugers Arch - Eur J Physiol DOI:10.1007/s00424-013-1302-4}

After the online publication of our article, we have been informed that our method of estimating the open probability of TRPM8 channel variants from the first derivative of the IV curve is theoretically incorrect and may result in considerably deviating values for the $\mathrm{V}_{50}$ and the slope after a fit to the Boltzmann equation. Accordingly, we have re-analyzed these values for the crucial variants with the conventional method, assessing the open probability from the conductance $\mathrm{G}=\mathrm{I} / \mathrm{V}$ (note that the reversal potential is practically $0 \mathrm{mV}$ ) and relating the conductance to the $\mathrm{G}_{\max }$ obtained after maximal stimulation with menthol at strongly depolarizing potentials.

The new values are found in the table below, listing the parameters derived from Boltzmann fits of activation curves $\left(G / G_{\max }\right.$ in dependence of $\left.\mathrm{V}\right)$. The apparent gating charge was calculated from the slope as $Z_{\text {app }}=\frac{R \cdot T}{F \cdot \text { slope }}$.

The online version of the original article can be found at http://dx.doi.org/ 10.1007/s00424-013-1302-4.

F. J. P. Kühn $(\bowtie) \cdot$ M. Winking • C. Kühn • D. C. Hoffmann •

A. Lückhoff

Institute of Physiology, Medical Faculty, RWTH Aachen,

D52057 Aachen, Germany

e-mail: fkuehn@physiology.rwth-aachen.de
The temperature was $273+29 \mathrm{~K}$. Data are mean \pm SEM $(n=4-7)$.

\begin{tabular}{lcll}
\hline TRPM8 variant & $\mathrm{V}_{50}(\mathrm{mV})$ & Slope $(\mathrm{mV})$ & $\mathrm{Z}_{\text {app }}\left(\mathrm{e}_{\mathrm{o}}\right)$ \\
Wild-type & $97 \pm 7$ & $56 \pm 10$ & $0.47 \pm 0.02$ \\
R842D+D802R & $90 \pm 4$ & $56 \pm 5$ & $0.47 \pm 0.01$ \\
D802R & $146 \pm 9$ & $46 \pm 7$ & $0.58 \pm 0.05$ \\
F839R+H845R & $153 \pm 11$ & $44 \pm 8$ & $0.68 \pm 0.03$ \\
F839R+H845R+T848K & $160 \pm 9$ & $41 \pm 6$ & $0.67 \pm 0.03$ \\
\hline
\end{tabular}

Most importantly for the conclusion of our paper, the rescue mutant R842D+D802R remains indistinguishable from wild-type TRPM8, although the crucial R842 has been removed from S4. If additional positively charged residues were introduced into either S3 (variant D802R) or into S4 (F839R+H845R and F839R+H845RT848K), significantly stronger depolarization is required for voltage-dependent activation. The slope values of the Boltzmann fits were marginally and unsystematically reduced by the positive charges, although a stronger reduction might be expected especially for the $\mathrm{S} 4$ mutants. The data support our view that voltage gating of TRPM8 should probably not be explained by a mobile positively charged voltage sensor in S4.

A more robust estimation of voltage sensitivity may require the limiting slope analysis that would be valid also in the case of non-Boltzmann behavior.

We apologize for any confusion we might have caused. 